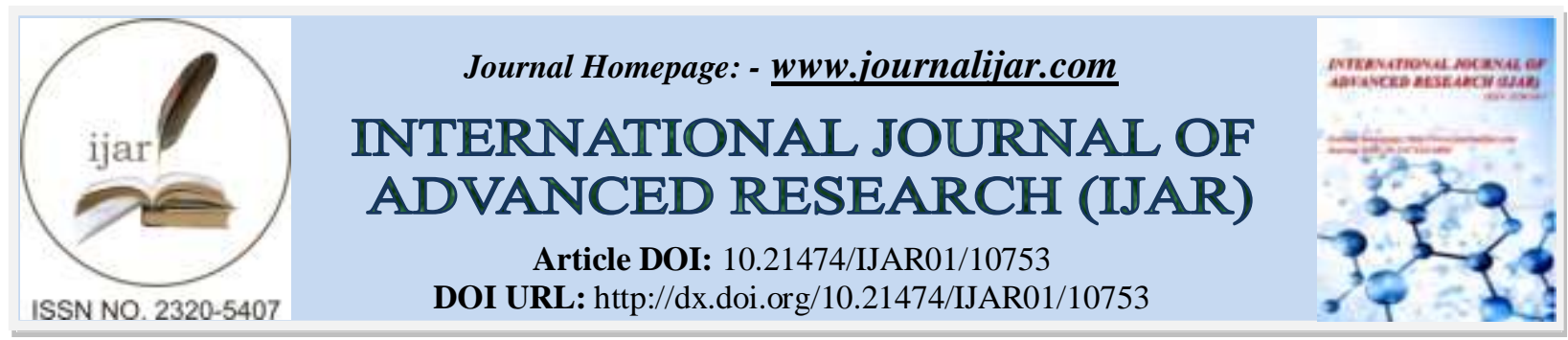

RESEARCH ARTICLE

\title{
A REVIEW ON LUPUS NEPHRITIES: PATHOGENESIS, CLASSIFICATION, AND MANAGEMENT
}

Balaga Rathnamala, K.Vishal, S.Salini and Enjum Nihith Chandra

\section{Manuscript Info}

Manuscript History

Received: 05 February 2020

Final Accepted: 07 March 2020

Published: April 2020

Keywords:-

Lupus Nephritis, Systemic Lupus

Erythematous, Immune Complexes,

Proteinuria, Cytokines

\section{Abstract}

Lupus nephritis is an autoimmune disease caused by systemic lupus erythematous, the prevalence of SLE and the chances of developing lupus nephritis (LN) vary considerably between different regions of the world and different races and ethnicities, a number of factors like genetic, environmental and immunology lead to inflammation of kidneys which is identified by biopsy, it is classified into six types based on the level of tissue damage and it is treated by induction, maintenance, adjuvant therapy and biological therapy. Mostly immunosuppressant's, antimalarials are used.

Copy Right, IJAR, 2020,. All rights reserved.

\section{Introduction:-}

\section{Definition:}

Lupus is an autoimmune disease in which one's own immune system attacks its cells and organs; lupus nephritis is inflammation of kidneys caused by systemic lupus erythematosus (SLE).

\section{Epidemiology:}

The prevalence of SLE and the chances of developing lupus nephritis (LN) vary considerably between different regions of the world and different races and ethnicities.A study of 208 cases of biopsy-proven lupus nephritis (176 women, 32 men): the overall prevalence was 4.4 per 100,000 population (95\% confidence interval [95\% CI] 3.85.0), 7.1 per 100,000 (95\% CI6.1-8.2) in women, and 1.4 per 100,000 (95\% CI 1.0-2.0) in men. The prevalence was significantly higher among women in the ethnic subgroups: 110.3 per 100,000population (95\% CI 55.0-197.3) in Chinese patients 99.2 per 100,000 (95\% CI 55.5-163.6) in Afro-Caribbean, 21.4 per 100,000 (95\% CI 12.0-35.2) in Indo-Asian (Asians from the Indian subcontinent), and 5.6 per100, 000 (95\% CI 4.7-6.7) in white patients. The overall annual incidence rate was 0.40 per 100,000 population per year $(95 \%$ CI $0.24-0.63)$, with a rate of 0.68 $\left(95 \%\right.$ CI0.40-1.10) in women and 0.09 (95\% CI 0.01-0.32) in men. ${ }^{(1)}$

\section{Etiology:}

There are multiple susceptible etiologies which results in abnormal immune responses, some of the factors are:

\section{Genetic Factors:}

Disease susceptibility genes associated with lupus nephritis

Programmed cell death: FAS, DNASE1, RIG1, ATG5, and MTMR3.

Immune complex clearance: FCGR[2A,2B,3A,3B], C1Q(A,B,C), C4(A,B), CRP, MBL2, CR1, ITGAM, IKZF1. Intra renal pathogenesis: TNFRSF1B, CCL2, CXCL8, CCR5, CXCL12, AGT, APOL1.

Adaptive immunity: HLA-DR, PTPN22, CTLA4.

Innate immunity: IFIH1, RIG1, MAVS, TREX1, MYD88, TRAF6, IRAK1, TNIP1.

\section{Corresponding Author:- Balaga Rathnamala}


Immunologic factors:

The presence of autoantibodies in patients, including anti-dsDNA, anti-SSA (Ro), anti-SSB (La), anti-Sm and antiRNPs, autoreactive B-lymphocytes.

Environmental factors:

UV light, Epstein-Barr virus smoking, alcohol, silica dust.

Clinical presentation of lupus nephritis ${ }^{[8]}$

1. proteinuria

2. hematuria

3. hypertension

4. Swelling of the legs, ankles and feet.

5. Less often, there can be swelling in the face or hands ${ }^{[7]}$

6. Weight gain

7. Dark urine

8. Frothy urine

9. The need to urinate during the night.

Prevalence of clinical manifestations in patients with lupus nephrities ${ }^{[12]}$ :

\begin{tabular}{lc} 
Clinical Manifestation & $\begin{array}{c}\text { Approximate } \\
\text { Prevalence, \% }\end{array}$ \\
\hline Proteinuria & 100 \\
Nephrotic range proteinuria/ & 50 \\
$\quad$ nephrotic syndrome & \\
Microscopic hematuria & 80 \\
Macroscopic hematuria & $<5$ \\
Urinary red blood cell casts & 30 \\
Other urinary cellular casts & 30 \\
Renal insufficiency & 60 \\
Rapid decline in kidney function & 15 \\
Hypertension & 30 \\
Tubular abnormalities & 70
\end{tabular}

\section{Pathogenesis of lupus nephritis:}

Extrarenal pathogenic mechanisms of lupus nephritis:

1. Impaired silent cell death and dead cell removal

2. Nuclear particles mimic viruses at viral recognition receptors of the innate immune system

3. Antiviral immunity

4. Autovaccination leading to persistent antinuclear antibody production

5. Flares triggered by transient autoantigen loads or unspecific immune activation 
A
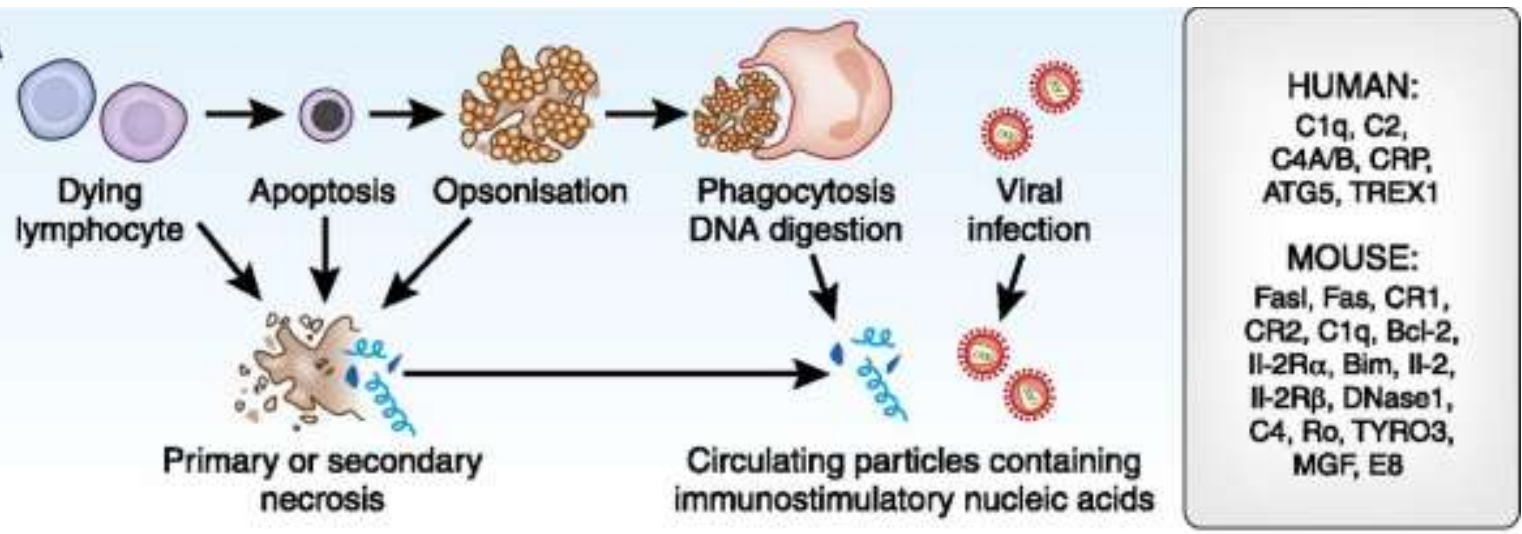

B
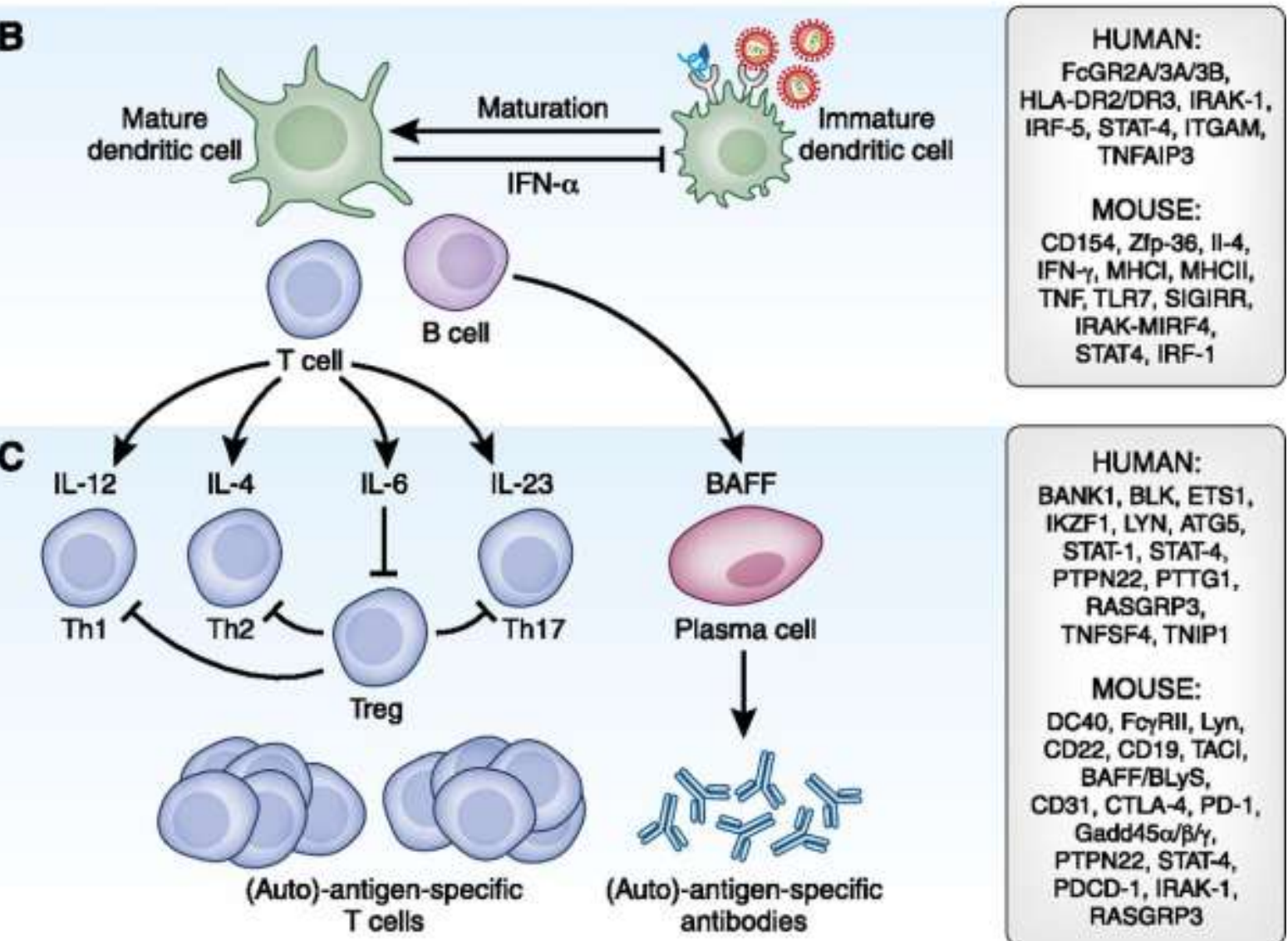

Intrarenal Pathomechanisms of SLE-Related Nephritis ${ }^{14\rfloor}$

Immune Complex (IC) Formation and Classical Complement Pathway Activation:

Circulating polyclonal autoantibodies bind to intrarenal nucleosomes and other autoantigens, which leads to local complement activation, cell injury, and subsequent cytokine and chemokine secretion.

The Immune Complex Formation Site Determines Lupus Nephritis Outcomes:

The polyclonal lupus autoantibody isotopes can localize to different compartments within the glomerulus, which affects the type of histopathological lesion as well as the alterations of glomerular function. Immune complex formation in the mesangium induces mesangioproliferative glomerulonephritis (lupus nephritis classes I and II), which is often mild and rarely progresses to end-stage kidney disease. Subendothelial immune complex formation (lupus nephritis classes III and IV) causes vascular obstruction by endothelial cell swelling and clotting, which promotes a decline of glomerular filtration rate. Vascular necrosis and glomerular basement membrane ruptures 
promote hematuria, crescent formation, and subsequently glomerulosclerosis. Subepithelial immune complex formation (membranous lupus nephritis class V) injures podocytes, which promotes massive proteinuria and podocyte loss-related glomerulosclerosis.

Induction of Cytokines, Chemokines, and Adhesion Molecules Recruits Leukocytes: Leukocyte recruitment amplifies intrarenal inflammation and promotes secondary tissue injury related to tissue inflammation and drives a vicious cycle of inflammation-induced tissue injury and injury-related inflammation.

Tertiary Lymphoid Organ Formation inside the Kidney:

Local expression of lymphotoxin and homeostatic chemokines drives tertiary lymphoid organ formation at sites of chronic inflammation to promote the (auto-) immune response, e.g. by local autoantibody production.

\section{Insufficient Regeneration and Tissue Scarring:}

Attempts to heal tissue injury often create new lesions. Lesions of hyperactive repair include hyperproliferation of mesangial cells (mesangioproliferative lupus nephritis), endothelial cells (endocapillary lupus nephritis), and parietal epithelial cells (crescentic lupus nephritis). Lesions of insufficient repair include podocyte loss-related scarring (glomerulosclerosis).

Environmental Triggers of SLE Activity ${ }^{[5]}$ :

Viral infections induce IFN- $\alpha$ release, which triggers antiviral immunity as well as lupus disease activity. Bacterial infections have a nonspecific immunostimulatory effect, which involves a transient expansion of autoreactive lymphocyte clones. Furthermore, bacterial products stimulate intra renal immune cells and renal cells, which can trigger a transient aggravation of proteinuria and kidney damage.

Drug-induced SLE involves inhibition of methyl-transferases, a process that enhances the unmasking of endogenous nucleic acids and the activation of TLR7 and TLR9. Progesterone and estrogens stimulate the sex hormonedependent immune regulatory pathways. Together, SLE develops from a peculiar combination of genetic variants that impair those mechanisms that normally prevent the exposure of nuclear particles to the immune system and their capacity to activate viral recognition nucleic acid receptors. The auto adjuvant activity of endogenous nucleic acids promotes an adaptive immune response against the components of the nuclear particle, a process identical to vaccination. This implies the expansion of $\mathrm{T}$ and $\mathrm{B}$ cell clones with specificities for predominantly nuclear autoantigens that account for the production of anti-nuclear antibodies, immune complex disease, and $\mathrm{T}$ celldependent tissue damage. Hormonal and environmental stimuli can enhance these processes at different levels.

Diagnosis: ${ }^{[9]}$

$\mathrm{X}$-rays of the kidneys

Blood tests ${ }^{[10]}$ :

If there is a loss of protein in the urine, a blood test can show if there is a lower level of protein in the blood. Blood tests can also show is there are imbalances of salt and water in the blood. Finally, blood tests can show the presence of antibodies-anti nuclear antibody, Antiphospholipid Antibodies, Anti-Sm, Anti-dsDNA, Anti-Ro(SSA) and Anti$\mathrm{La}(\mathrm{SSB})$, that are typically high in persons who have lupus nephritis. Anti-C1q antibody (Ab) titers have been elevated in patients with lupus with renal involvement ${ }^{[6]}$

Urine tests: A urinalysis will check for the presence of red and white blood cells in the urine or high levels of Urine Protein/Microalbuminuria and Creatinine Clearance

Imaging studies: Two of these types of studies - an intravenous pyelogram and a sonogram - are usually done before a kidney biopsy. In an intravenous pyelogram, the dye is injected into the body and collects in the kidneys. An X-ray is taken that shows the outline of the kidneys with the dye. A sonogram uses soundwaves transmitted through the body and their echoes to show the shape and size of the surfaces of the kidney.

A biopsy of the kidney. This test involves taking a sample of kidney tissue to examine under a microscope. If blood and urine studies suggest lupus nephritis, a biopsy is done to confirm the diagnosis. A biopsy can also help find out how widespread and severe the kidney disease is, A biopsy is most often done by inserting a narrow needle through the skin of the back and removing a small piece of kidney. 
Classification of lupus nephritis ${ }^{[11]}$ :

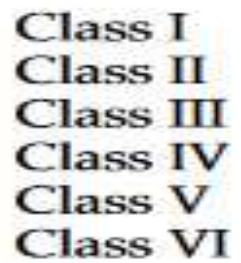

\author{
Minimal mesangial L.N \\ Mesangial proliferative LN \\ Focal LN ( $<50 \%$ of glomeruli) ${ }^{a}$ \\ Diffuse LN $\left(\geq 50 \%\right.$ of glomeruli) ${ }^{a, b}$ \\ Membranous LN \\ Advanced sclerotic LN $\mathbf{9 0 \%}$ of \\ glomeruli globally sclerosed \\ without residual activity)
}

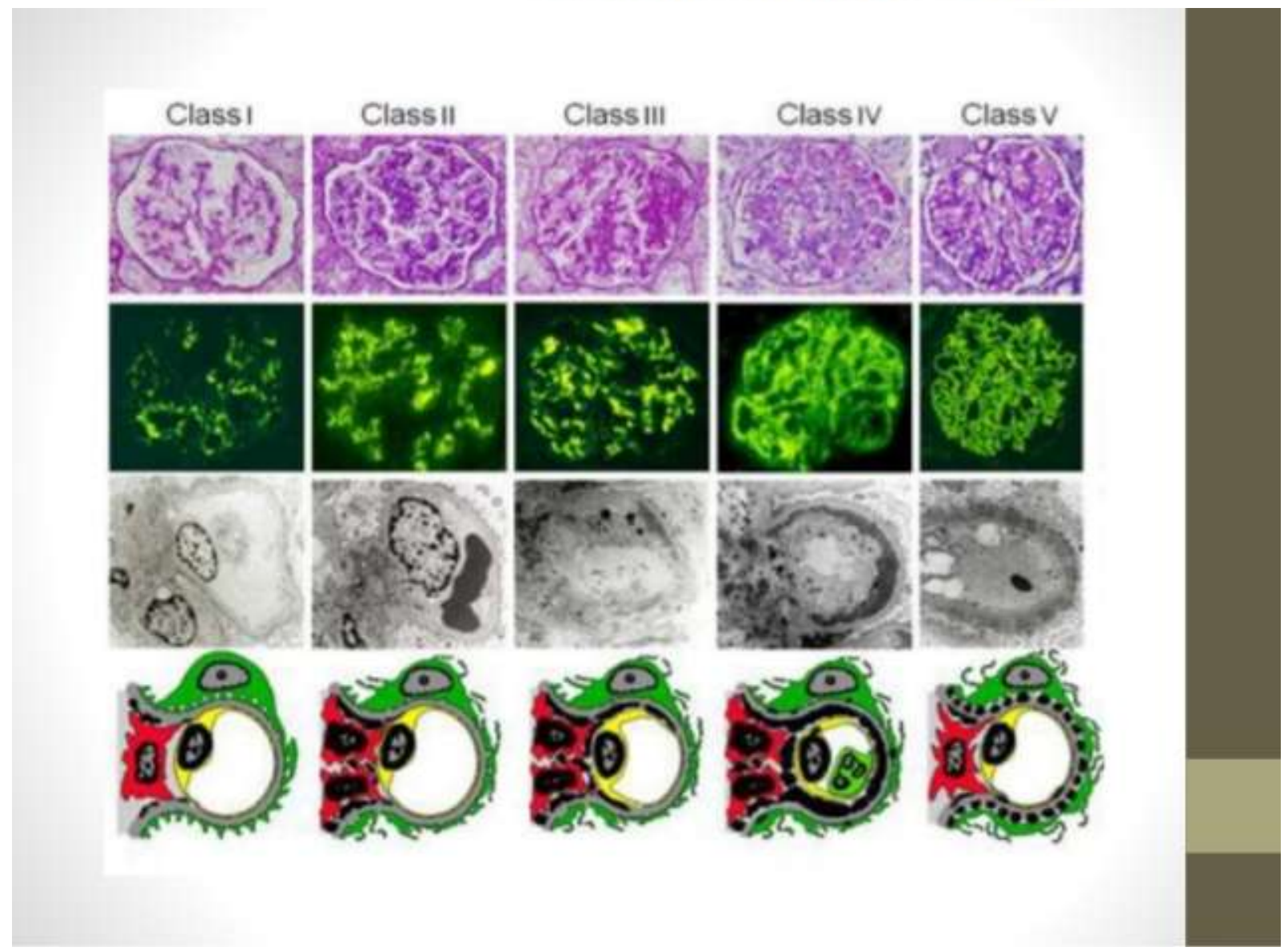

Class I (minimal mesangial LN) and Class II (mesangial proliferative LN) ${ }^{[3]}$ :

The high regenerative capacity of mesangial cells, mesangial expansion does not progress and usually does not cause proliferative or sclerosing glomerular injury. Disease class I includes early glomerular involvement with minimal mesangial tissue injury mediated by IC. In LN class II, injury mediated by IC is accompanied by hypercellularity and mesangial expansion. These classes are associated with a good prognosis. Treatment with immunosuppressants is generally recommended to manage extrarenal manifestations.

Class III (focal proliferative LN) and Class IV (diffuse proliferative LN) :

Proliferative LN (classes III and IV) is caused by the deposition of IC in the subendothelial space of the glomerular capillaries, either alone or in combination with the deposition of IC in the mesangial region. Sub endothelial deposition triggers the production of IFN-gamma by endothelial cells and, consequently, local inflammation and endocapillary hypercellularity. Reticular aggregates - ultrastructural findings characteristically seen in scenarios of elevated IFN-gamma secretion - may also form. Severe modes of the disease have been associated with crescentic formations stemmed from the rupture of glomerular capillary loops and leakage of mitogenic proteins (mainly 
fibrinogen) into the urinary space, with subsequent involvement of the parietal epithelium. Proliferative LN presents lesions that characterize activity and chronicity.

The criteria for activity are endocapillary hypercellularity; glomerular neutrophils/karyorrhexis; fibrinoid necrosis; wire loop lesions and/or hyaline thrombi in the glomeruli; cellular and/or fibro cellular crescents; and interstitial inflammation.

The criteria for chronicity include: total score of segmental or global glomerulosclerosis; fibrous crescents; tubular atrophy and interstitial fibrosis

Involvement with active (A) and/or chronic (C) lesions in less than $50 \%$ of the glomeruli is seen in LN class III. Involvement of more than $50 \%$ of the glomeruli indicates LN class IV, which is subdivided into "S" - segmental glomerular injury ${ }^{[13]}$, i.e., injuries affecting less than half of the glomerular tufts - and "G" - global glomerular injury, i.e., injuries affecting more than half of the glomerular tufts.

Although other injuries may occur with LN, they are not used for classification purposes. Nevertheless, they may affect the choice of treatment.

\section{Tubulointerstitial injury:}

clonal expansion of B cells and plasma cells may trigger local production of antibodies and consequent increases in inflammatory response and formation of tertiary lymphoid tissue. The Deposition of IC along the tubular basement membrane also occurs. These injuries may help identify patients responsive to therapies targeting B cells, such as treatment with rituximab.

Vascular injuries are common and may affect patient prognosis. They originate from the deposition of IC in vascular smooth muscle cells and endothelial cells or by local complement activation. Five types of vascular injuries are often observed: vascular IC deposits, arterio nephrosclerosis, thrombotic microangiopathy, noninflammatory necrotizing vasculopathy, and vasculitis. Other possible events include endothelial edema, transmural vasculitis with fibrinoid necrosis, mesangiolysis or fibrin thrombi and, enlargement of the lamina rara interna of the glomerular basement membrane seen with the aid of electron microscopy. Some of these injuries may be related to manifestations of LN, including systemic hypertension, dyslipidemia, and thromboembolism. Vascular injuries may help identify patients potentially responsive to eculizumab and thrombomodulin.

Podocyte injuries are common and stem from the loss of expression of the proteins present in the slit diaphragm (nephrin and podocin) and the disorganization of the podocyte cytoskeleton, culminating with the flattening, effacement, and microvillus transformation of the foot processes. Affected patients develop marked proteinuria. Podocyte injuries may be used to identify patients potentially responsive to calcineurin inhibitors.

Crescentic injuries arise from immune deposits or direct attack by inflammatory cells. Between 30-100\% of the patients with diffuse crescentic injury are positive for ANCA and/or anti-myeloperoxidase antibodies, showing overlapping SLE and ANCA-positive vasculitis. This group of injuries may help identify patients potentially responsive to plasmapheresis and monoclonal anti-C5aR antibody.

\section{Class V (membranous LN):}

LN class V originates from the subepithelial IC deposition of either immune complexes transiting through the glomerular basement membrane of immune complexes formed locally to deal with podocyte antigens. The complement system is then activated locally, usually with the formation of membrane attack complex (C5b-9), thickening of the glomerular basement membrane, and destabilization of the podocyte cytoskeleton..LN class $\mathrm{V}$ is often associated with nephrotic-range proteinuria with or without hematuria. This class of the disease may occur in association with proliferative LN (Class III or IV). As class V evolves to chronicity, there is typically the development of segmental or global glomerulosclerosis, without the super-imposition of proliferative lupus nephritis $^{[14]}$.

Class VI (advanced sclerosing LN):

LN class VI results from the progression of lupus nephritis. In this disease class, glomerular, vascular, and tubule interstitial injuries from glomerulosclerosis are seen in more than $90 \%$ of the analyzed glomeruli. 
Therapies used in the treatment of lupus nephritis ${ }^{[15]}$

Patients who present with class I or class II mesangial disease generally have good long-term outcomes, and treatment with either an angiotensin-converting enzyme (ACE) inhibitor or angiotensin receptor blocker (ARB) is recommended for any patient with proteinuria $\geq 0.5 \mathrm{~g} /$ day, with the primary goal being the prevention of the progression of nephritis.

Focal or diffuse lupus nephritis (classes III-IV) confers a much greater risk of progression, potentially to ESRD, and requires more aggressive treatment with immunosuppressive medications. Treatment regimens generally include an aggressive induction phase in which the goal is to induce complete or partial disease remission over the course of a few months. This is followed by a maintenance phase in which lower doses of immunosuppressive agents are used to maintain remission and prevent flare-ups. antimalarials may reduce the risk of renal flares, improve maintenance of remission, and reduce the risk of progression to ESRD in addition to reducing the risk of thrombosis. The current ACR guidelines recommend the addition of hydroxychloroquine to the regimens of all SLE patients with nephritis

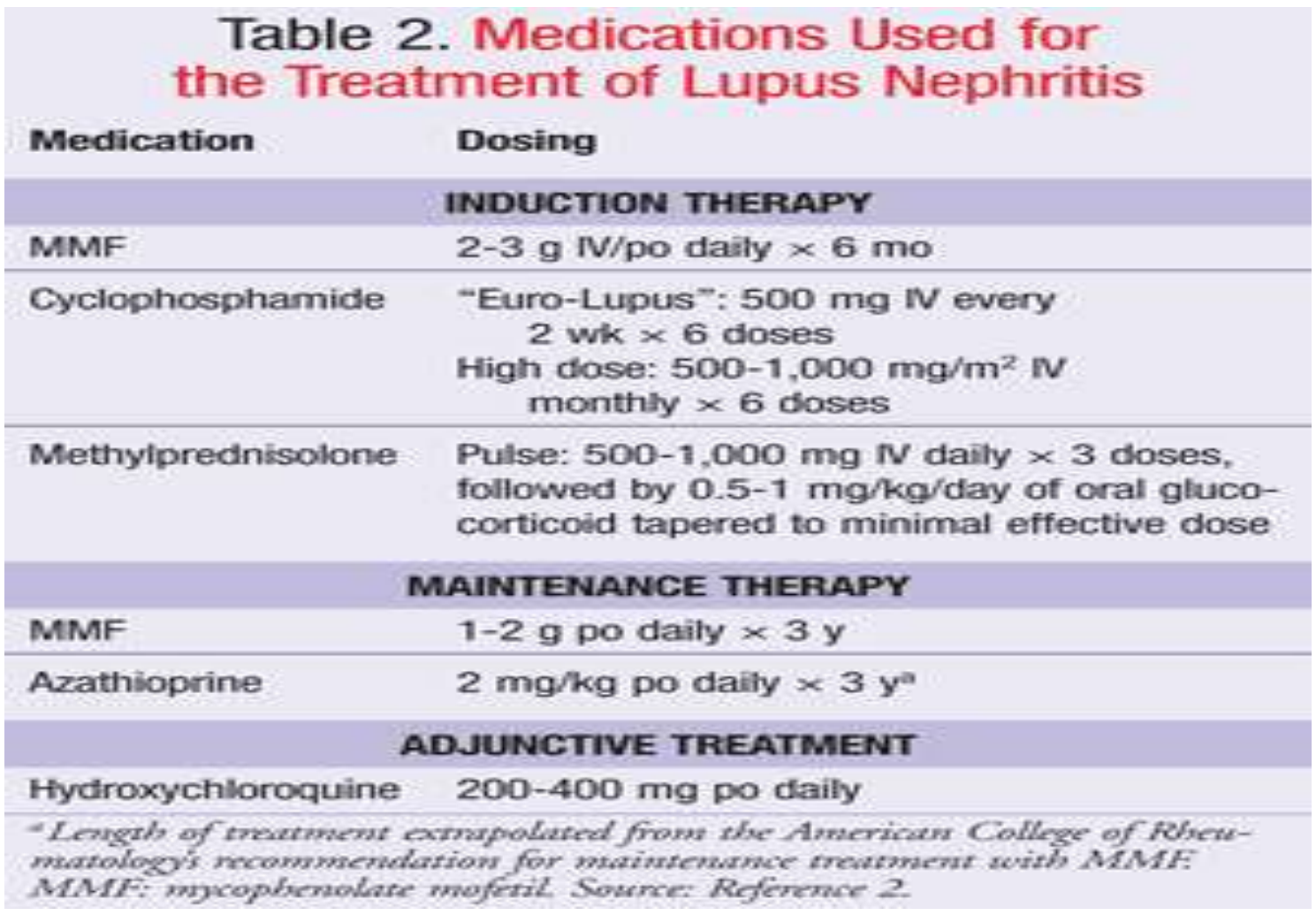

\section{Cyclophosphamide Induction Therapy:}

Cyclophosphamide, a synthetic antineoplastic drug chemically related to the nitrogen mustards, It exerts its antineoplastic and immunosuppressant effects by cross-linking DNA preferentially in quickly dividing cells, such as cancerous cells and leukocytes.

The combination of cyclophosphamide and methylprednisolone as induction therapy has been effective than corticosteroids alone. Daily oral cyclophosphamide may also be used for induction therapy; however, IV pulse therapy is preferred due to the decreased cumulative exposure to cyclophosphamide reduces the incidence of cytopenia. Amenorrhoea, infertility, and opportunistic infections such as herpes zoster While this combination is effective, patients treated with cyclophosphamide and methylprednisolone for induction therapy in lupus nephritis are at an increased risk of adverse drug events as compared to those treated with methylprednisolone monotherapy. Adverse events include amenorrhea cervical dysplasia, herpes zoster, and infection.

Lower-dose cyclophosphamide for induction therapy: traditional high-dose therapy, dosed monthly for 6 months followed by quarterly dosing $\left(0.5 \mathrm{~g} / \mathrm{m}^{2}\right.$ initially, then adjusted based on white blood cell count nadir), with low-dose 
therapy (500 mg every 2 weeks $\times 6$ doses followed by azathioprine maintenance therapy of $2 \mathrm{mg} / \mathrm{kg} / \mathrm{day}$ ) has reduced adverse effects.

\section{Induction Therapy With Mycophenolate mofetil (MMF):}

MMF is metabolized to mycophenolic acid, which inhibits inosine monophosphate dehydrogenase and in turn inhibits the de novo pathway for guanine nucleotide synthesis. As the proliferation of $\mathrm{B}$ and $\mathrm{T}$ cells is highly dependent on this pathway, MMF has a potent cytostatic effect on lymphocytes.

oral MMF ( $2 \mathrm{~g}$ daily for 6 months followed by $1 \mathrm{~g}$ daily for 6 months) or oral cyclophosphamide $(2.5 \mathrm{mg} / \mathrm{kg} / \mathrm{day}$ for 6 months) followed by oral azathioprine $(1.5 \mathrm{mg} / \mathrm{kg} / \mathrm{day}$ for 6 months). oral prednisolone daily. Infections were significantly less common in the MMF

MMF is an alternative to cyclophosphamide induction therapy, and may confer less risk of toxicity ${ }^{[18]} .2$ to $3 \mathrm{~g} /$ day of MMF as part of an induction regimen with pulsed corticosteroids. The $3 \mathrm{~g} /$ day dosage is favored in patients with proteinuria and significant rises in serum creatinine.

\section{Maintenance Therapy:}

Once remission has been induced, long-term maintenance therapy should be initiated to reduce the risk of recurrence and long-term complications of the disease. MMF-1-2g/po for 3 years. Azathioprine (AZA) is a purine analog which inhibits DNA synthesis and acts most strongly on rapidly proliferating cells. A dose of $2 \mathrm{mg} / \mathrm{kg} /$ day for 3 years is preferred. AZA is preferred as maintenance therapy, in pregnant patients and in patients intolerant to other first-line induction agents.however; doses should be tapered over time to reduce the risk of adverse reactions.

\section{Adjuvant therapy:}

Hydroxychloroquine (HCQ) is an antimalarial drug with anti-inflammatory, antithrombotic and immunomodulatory properties. The use of HCQ has been associated with decreased probability of LN when used before the onset of LN in SLE ${ }^{[57]}$ and also retards the onset of renal damage in patients with $\mathrm{LN}^{[58]}$ The use of HCQ has also been associated with increased probability of remission, reduced frequency of flares and improved survival.

\section{Others:}

Angiotensin inhibitors/blockers [angiotensin-converting enzyme inhibitor/angiotensin receptor blocker (ACEI/ARB)] are effective in reducing proteinuria in diabetic nephropathy and other proteinuric glomerular diseases. use of ACEI/ARB was found to be effective in reducing proteinuria and improving serum albumin ${ }^{[59,60]}$ .Their use was also associated with retarding the occurrence of renal involvement and reducing overall disease activity in $\operatorname{SLE}^{[61] .}$

\section{Membranous Lupus Nephritis Treatment:}

The treatment of membranous lupus nephritis, ISN/RPS class V, differs from that of proliferative nephritis. Milder disease with stable kidney function, subnephrotic proteinuria, and the absence of proliferative lesions may only require treatment with an $\mathrm{ACE}$ inhibitor or an $\mathrm{ARB}$ in order to suppress the renin-angiotensin-aldosterone system (RAAS). For a disease that is more severe or includes proliferative lesions, studies have demonstrated that treatment with a corticosteroid in combination with either cyclosporine, MMF, or cyclophosphamide is an effective regimen ${ }^{[34,33]}$ The first-line treatment with MMF and prednisone followed by maintenance therapy with MMF or azathioprine. 


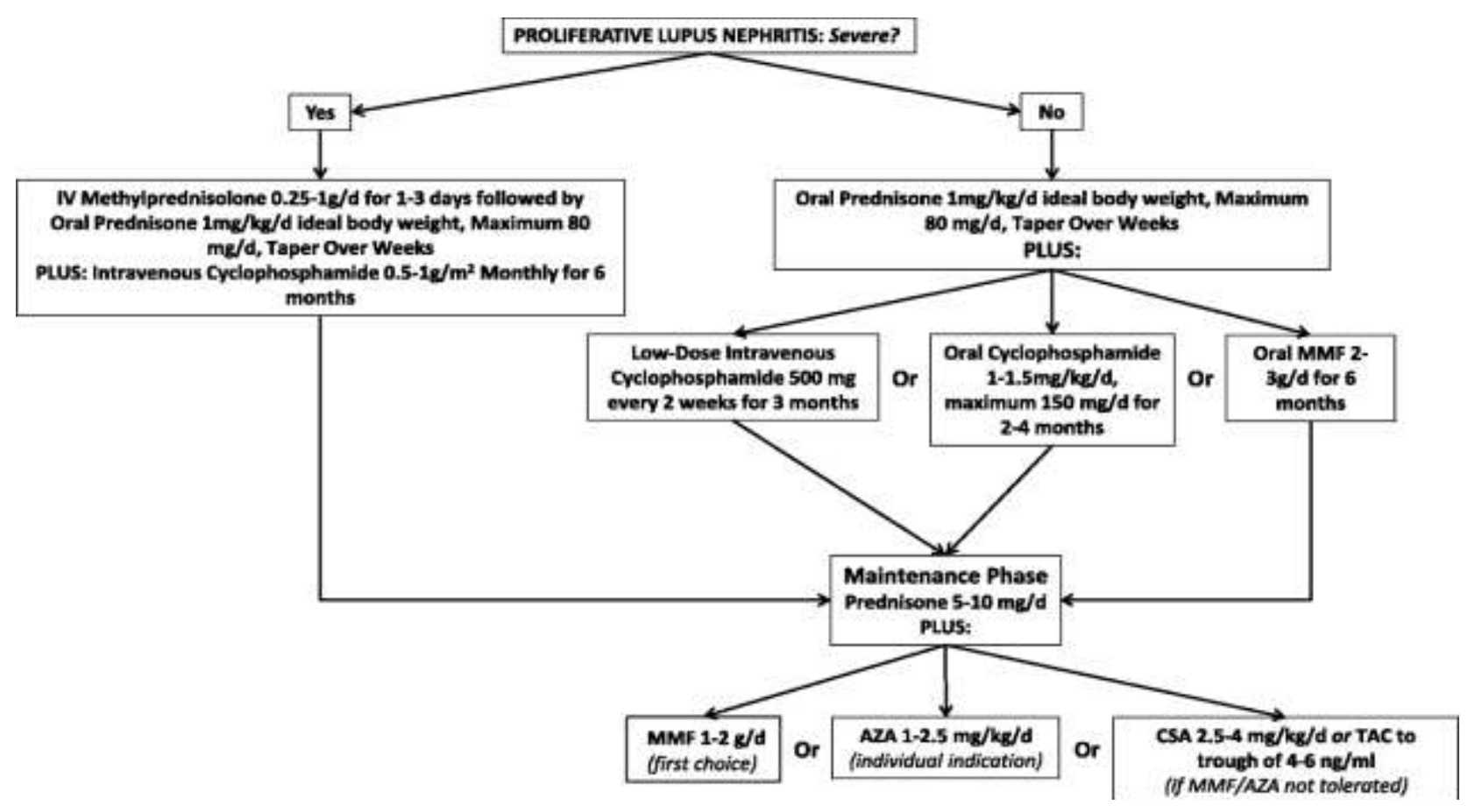

Calcineurin inhibitors (CNIs) ${ }^{[16]}$ :

Calcineurin inhibitors are a class of immunosuppressant drugs that decrease lymphocytic proliferation through the inhibition of a phosphatase calcineurin, CNIs have got a dual mode of action viz., immunosuppression and stabilization of podocyte cytoskeleton ${ }^{[62]}$ Tacrolimus (TAC) has been found to be effective in proliferative, membranous as well as resistant $\mathrm{LN}$ and may have a role in pregnant patients ${ }^{[63]}$ Although effective in LN, CNI has been invariably associated with the risk of relapse and always carries risk of nephrotoxicity with long-term use. Lower CNI levels may be effective in LN.

Biological agents in lupus nephritis:

The various biological agents consisted of anti-B-cell therapies targeting either B-cell surface antigens (anti-CD20 and anti-CD22) or B-cell survival factors [anti-B lymphocyte stimulator/A proliferation-inducing ligand (antiBLyS/APRIL) monoclonal antibodies], anti-cytokines antibodies (anti-interleukin-6) and novel drugs intervening in B-T cell co-stimulation [cytotoxic T-lymphocyte-associated protein 4 (CTLA4-Ig) The anti-B-cell-targeted therapies that have been investigated are rituximab (RTX) ${ }^{[64]}$ [chimeric anti-CD20 monoclonal antibody (MAB)], ocrelizumab $^{[65]}$ (humanized anti-CD20 MAB), epratuzumab ${ }^{[66]}$ (anti-CD 22 humanized MAB) and belimumab ${ }^{[67]}$ [Fully human anti-BlyS (B lymphocyte stimulator) MAB]. Other targeted therapies investigated included abatacept $^{[68]}$ [(CTLA4-Ig) fusion protein] and atacicept ${ }^{[69]}$ (soluble fully human recombinant anti-APRIL fusion protein).

Non-pharmacological therapy ${ }^{[17]}$ :

1. Drink enough fluids to stay well hydrated.

2. Eat a low-sodium diet, especially if hypertension is an issue.

3. Avoid smoking and drinking alcohol.

4. Exercise regularly.

5. Maintain healthy blood pressure.

6. Limit cholesterol.

7. Avoid medications that can affect the kidneys, such as nonsteroidal anti-inflammatory drugs (NSAIDs).

8. avoid over-exposure to sunlight with the use of adequate sunscreen protection

9. avoiding "live" vaccination if on immunosuppressive agents

10. adherence to a diet low in saturated fat and high in fish oil

11. stress avoidance 


\section{Conclusion:-}

There is more uncertainty about how to treat lupus nephritis.

\section{References:-}

1. 1.Mumtaz Pate ${ }^{1}$, Alexandra M. Clarke, ${ }^{2}$ Ian N. Bruce, ${ }^{2}$ and Deborah P. M. Symmons ${ }^{2}$,The Prevalence and Incidence of Biopsy-Proven Lupus Nephritis in the UK, ARTHRITIS \& RHEUMATISM Vol. 54, No. 9, September 2006, pp 2963-2969DOI 10.1002/art.22079@ 2006, American College of Rheumatology.

2. Melissa E. Munroe ${ }^{1}$ and Judith A. James ${ }^{1,2,{ }^{*}}$ Genetics of Lupus Nephritis: Clinical Implications, Semin Nephrol. Author manuscript; available in PMC 2016 Sep 1.Published in final edited form as: Semin Nephrol. 2015 Sep; 35(5): 396-409. doi: 10.1016/j.semnephrol.2015.08.002 PMCID: PMC4653095 NIHMSID: NIHMS720613PMID: 26573543

3. Brazilian Journal of Nephrology, Print version ISSN 0101-2800On-line version ISSN 2175-8239, J. Bras. Nefrol. vol.41 no.2 São Paulo Apr./June 2019 Epub Nov 14, 2018 https://doi.org/10.1590/2175-8239-jbn-20180097

4. Yujuan Liu Hans-Joachim Anders Nephrologisches Zentrum, Medizinische Klinik und Poliklinik IV, Klinikum der Universität, München, Munich, GermanyLupus Nephritis: From Pathogenesis to Targets for Biologic Treatment, Nephron Clin Pract 2014;128:224-231DOI: 10.1159/000368581

5. Maciej Lech and Hans-Joachim AndersDepartment of Nephrology, Medical Clinic and Polyclinic IV, University of Munich, Munich, GermanyThe Pathogenesis of Lupus Nephritis j Am Soc Nephrol24:1357-1366, 2013 ISSN : 1046-6673/2409-1357

6. Gabriella Moroni MD, etal :Anti-C1q antibodies may help in diagnosing a renal flare in lupus nephritis, American Journal of Kidney Diseases, Volume 37, Issue 3, March 2001, Pages 490-498

7. WebMD Medical Reference Reviewed by David Zelman, MD on August 12, 2019

8. Chi Chiu Mok : Understanding lupus nephritis: diagnosis, management, and treatment options, International Journal of Women's Health, Int J Womens Health. 2012; 4: 213-222. Published online 2012 May 22. doi: 10.2147/IJWH.S28034,PMCID: PMC3367406, PMID: 22675266

9. CEDARS-SINAI, Lupus Nephritis, (C) 2019 Cedars-Sinai. All Rights Reserved. A 501(c)(3) non-profit organization. www.cedars.sinai.edu

10. webmed, labtest for lupus, www.webmed.com.

11. Giovanna Giannico and Agnes B. Fogo, Lupus Nephritis: Is the Kidney Biopsy CurrentlyNecessary in the Management of Lupus Nephritis?, Copyright (C) 2013 by the American Society of Nephrology, www.cjasn.org Vol 8 January, 2013

12. Salem Almaani, Alexa Meara, and Brad H. Rovin, Glomerular DiseasesUpdate on Lupus Nephritis, clinical Journal of the American Society of Nephrology, CJASN.ePressPublished on November 7, 2016 as doi: 10.2215/CJN.05780616

13. GS Markowitzland VD D'Agati11Department of Pathology, College of Physicians and Surgeons, Columbia University, New York, New York, USA, The ISN/RPS 2003 classification of lupus nephritis:An assessment at 3 years, Kidney International(2007)71, 491-495, http://www.kidney-international.org

14. JANJ. WEENING etal, The classification of glomerulonephritis in systemic lupuserythematosus revisited, Kidney International, Vol. 65 (2004), pp. 521-530

15. Zachary Heeter, autoimmune Current Therapies for Lupus Nephritis, US pharmacist resource for excellence, Published June 20, 2012, www.uspharmacist.com

16. Ajay Jaryal, Sanjay Vikrant : Department of Nephrology, Indira Gandhi Medical College (IGMC), Shimla, India, Current status of lupus nephritis, Indian journal of medical research, Year : 2017 / Volume : 145 / Issue : 2/ Page : 167-178.

17. Ioannou $\mathrm{Y}^{1}$, Isenberg DA, Current concepts for the management of systemic lupus erythematosus in adults: a therapeutic challenge. Postgrad Med J. 2002 Oct;78(924):599-606. www.ncbi.nlm.nih.gov

18. Christian Burchardi; Detlef Schlöndorff, Induction Therapy for Active Lupus Nephritis: Mycophenolate Mofetil Versus Cyclophosphamide, Nature Clinical Practice Nephrology, Nat Clin Pract Nephrol. 2006;2(6):314-315.

19. 19.Chan $\mathrm{TM}^{1}$ Lupus nephritis: induction therapy,2005;14 Suppl 1:s27-32. PMID: 15803928, DOI:10.1191/0961203305lu2114oa

20. Lawrence H Brent, Arati S Karhadkar, Lupus Nephritis Workup,Updated: Mar 04, 2019, www.medscape.com

21. Saba Kiremitci * and Arzu EnsarClassifying Lupus Nephritis: An Ongoing StoryScientificWorldJournal. 2014; 2014: 580620, Published online 2014 Dec 8. doi: 10.1155/2014/580620, PMCID: PMC4274910, PMID: 25548784 
22. Rosalie M. Sterner, ${ }^{1,2}$ Stella P. Hartono, ${ }^{1,2}$ and Joseph P. Grande ${ }^{1, *}$ The Pathogenesis of Lupus Nephritis, J Clin Cell Immunol. 2014 Apr; 5(2): 205. Published online 2014 Apr. doi: 10.4172/2155-9899.1000205

23. Schwartzman-Morris J, Putterman C: Gender differences in the pathogenesis and outcome of lupus and of lupus nephritis. Clin Dev Immunol 2012: 604892, 2012

24. Maciej Lech and Hans-Joachim Anders :The Pathogenesis of Lupus Nephritis, JASN September 2013, 24 (9) 1357-1366; DOI: https://doi.org/10.1681/ASN.2013010026

25. Rebecca Wing-Yan Chan etal : Intrarenal cytokine gene expression in lupus nephritis,BMJ Annals of the Rheumatic Diseases http://dx.doi.org/10.1136/ard.2006.063123

26. van Bruggen MC etal: Pathophysiology of lupus nephritis: the role of nucleosomes. The Netherlands Journal of Medicine, 01 Dec 1994, 45(6):273-279, PMID: 7838243

27. Hans-Joachim Anders ${ }^{1}$, Brad Rovin ${ }^{2}$ :A pathophysiology-based approach to the diagnosis and treatment of lupus nephritis, ISN DOI: https://doi.org/10.1016/j.kint.2016.05.017

Liu Y. - Anders H.-J : Lupus Nephritis: From Pathogenesis to Targets for Biologic Treatment, Nephron Clin Pract 2014;128:224-231 https://doi.org/10.1159/000368581

29. 29. Alberto de Zubiria Salgado ${ }^{1,2}$ and Catalina Herrera-Diaz ${ }^{2}$ : Lupus Nephritis: An Overview of Recent Findings, auto immune disease, Volume 2012 |Article ID 849684 | 21 pages | https://doi.org/10.1155/2012/849684

30. Hahn BH, McMahon MA, Wilkinson A, et al. American College of Rheumatology guidelines for screening, treatment, and management of lupus nephritis. Arthritis Care Res. 2012;64:797-808

31. Houssiau FA. Cyclophosphamide in lupus nephritis. Lupus. 2005;14:53-58

32. Houssiau FA, Vasconcelos C, D'Cruz D, et al. Immunosuppressive therapy in lupus nephritis: the Euro-Lupus Nephritis Trial, a randomized trial of low-dose versus high-dose intravenous cyclophosphamide. Arthritis Rheum. 2002;46:2121-2131

33. Austin HA 3rd, Ilei GG, Braun MJ, Ballow JE. Randomized, controlled trial of prednisone, cyclophosphamide, and cyclosporine in lupus membranous nephropathy. J Am Soc Nephrol. 2009;20:901-911.

34. Radhakrishnan J, Moutzouris DA, Ginzler EM, et al. Mycophenolate mofetil and intravenous cyclophosphamide are similar as induction therapy for class V lupus nephritis. Kidney Int. 2010;77:152-160.

35. Liu Z, Davidson A: Taming lupus-a new understanding of pathogenesis is leading to clinical advances. Nat Med 18: 871-882, 2012

36. Saxena R, Mahajan T, Mohan C: Lupus nephritis: Current update. Arthritis Res Ther 13: 240, 2011

37. Muñoz LE, Lauber K, Schiller M, Manfredi AA, Herrmann M: The role of defective clearance of apoptotic cells in systemic autoimmunity. Nat Rev Rheumatol 6: 280-289, 2010

38. Lande R, Ganguly D, Facchinetti V, Frasca L, Conrad C, Gregorio J, Meller S, Chamilos G, Sebasigari R, Riccieri V, Bassett R, Amuro H, Fukuhara S, Ito T, Liu YJ, Gilliet M: Neutrophils activate plasmacytoid dendritic cells by releasing self-DNA-peptide complexes in systemic lupus erythematosus. Sci Transl Med 3: 73ra19, 2011

39. Caricchio R, McPhie L, Cohen PL: Ultraviolet B radiation-induced cell death: Critical role of ultraviolet dose in inflammation and lupus autoantigen redistribution. J Immunol 171: 5778-5786, 2003

40. Yung S, Chan TM: Autoantibodies and resident renal cells in the pathogenesis of lupus nephritis: Getting to know the unknown. Clin Dev Immunol 2012: 139365, 2012

41. Cameron JS: Systemic lupus erythematosus. In: Immunologic Renal Disease, edited by Nielson EG, Couser WG, Philadelphia, Lippincott-Raven, pp 1055-1094,1997

42. Tan EM, Cohen AS, Fries JF: The 1982 revised criteria for the classification of systemic lupus erythematosus. Arthritis Rheum25 : 1276-1282,1982

43. Donadio JV, Glassock RJ: Immunosuppressive drug therapy in lupus nephritis. Am J Kidney Dis 21:239 -250, 1993

44. Cameron JS: What is the role of long-term cytotoxic agents in the treatment of lupus nephritis? J Nephrol6 : $172-176,1993$

45. Ginzler E, Sharon E, Diamond H, Kaplan D: Long-term maintenance therapy with azathioprine in systemic lupus erythematosus. Arthritis Rheum 18: 27-34,1975

46. Walport MJ: The pathogenesis of systemic lupus erythematosus. In:Oxford Textbook of Clinical Nephrology, 2nd Ed., edited by Davison AM, Cameron JS, Grünfeld J-P, Kerr DNS, Ritz E, Winearls CG, London, Oxford University Press, 1997, pp917 -935

47. Elena V. Zakharova etal : Immunosuppressive Treatment for Lupus Nephritis, Volume 2016 |Article ID 7407919| 7 pages | https://doi.org/10.1155/2016/7407919 
48. H. S. Koo, S. Kim, and H. J. Chin, "Remission of proteinuria indicates good prognosis in patients with diffuse proliferative lupus nephritis," Lupus, vol. 25, no. 1, pp. 3-11, 2016.

49. Chan TM, Li FK, Tang CS, et al. Efficacy of mycophenolate mofetil in patients with diffuse proliferative lupus nephritis. N Engl J Med. 2000;343:1156-1162.

50. Felson DT, Anderson J: Evidence for the superiority of immunosuppressive drugs and prednisone over prednisone alone in lupus nephritis. N Engl J Med 311:1528 -1533, 1984

51. Boumpas DT, Austin HA III, Vaughan EM, Klippel JH, Steinberg AD, Yarboro CH, Balow JE: Controlled trial of pulse methylprednisolone versus two regimens of pulse cyclophosphamide in severe lupus nephritis.Lancet 340:741 -745, 1992

52. Bansal VK, Beto JA: Treatment of lupus nephritis: A metaanalysis of clinical trials. Am J Kidney Dis 29:193 199, 1997

53. Parikh SV, Rovin BH. Current and Emerging Therapies for Lupus Nephritis. J Am Soc Nephrol 2016; 27:2929.

54. Bomback AS, Appel GB. Updates on the treatment of lupus nephritis. J Am Soc Nephrol 2010; 21:2028.

55. Richardson B, Scheinbart L, Strahler J, Gross L, Hanash S, Johnson M: Evidence for impaired T cell DNA methylation in systemic lupus erythematosus and rheumatoid arthritis. Arthritis Rheum 33: 1665-1673, 1990

56. Cornacchia E, Golbus J, Maybaum J, Strahler J, Hanash S, Richardson B: Hydralazine and procainamide inhibit T cell DNA methylation and induce autoreactivity. J Immunol 140: 2197-2200, 1988

57. Sisó A, Ramos-Casals M, Bové A, Brito-Zerón P, Soria N, Muñoz S, et al. Previous antimalarial therapy in patients diagnosed with lupus nephritis: Influence on outcomes and survival. Lupus 2008; 17 : 281-8.

58. Pons-Estel GJ, Alarcón GS, McGwin G Jr., Danila MI, Zhang J, Bastian HM, et al. Protective effect of hydroxychloroquine on renal damage in patients with lupus nephritis: LXV, data from a multiethnic US cohort. Arthritis Rheum 2009; $61: 830-9$.

59. Tse KC, Li FK, Tang S, Tang CS, Lai KN, Chan TM. Angiotensin inhibition or blockade for the treatment of patients with quiescent lupus nephritis and persistent proteinuria. Lupus 2005; 14 : 947-52.

60. Kitamura N, Matsukawa Y, Takei M, Sawada S. Antiproteinuric effect of angiotensin-converting enzyme inhibitors and an angiotensin II receptor blocker in patients with lupus nephritis. J Int Med Res 2009; 37 : 892 8.

61. Durán-Barragán S, McGwin G Jr., Vilá LM, Reveille JD, Alarcón GS; LUMINA (LIX): A multiethnic US cohort. Angiotensin-converting enzyme inhibitors delay the occurrence of renal involvement and are associated with a decreased risk of disease activity in patients with systemic lupus erythematosus - results from LUMINA (LIX): A multiethnic US cohort. Rheumatology (Oxford) 2008; 47 : 1093-6.

62. Faul C, Donnelly M, Merscher-Gomez S, Chang YH, Franz S, Delfgaauw J, et al. The actin cytoskeleton of kidney podocytes is a direct target of the antiproteinuric effect of cyclosporine A. Nat Med 2008; $14: 931-8$

63. Gordon S, Denunzio T, Uy A. Success using tacrolimus in patients with proliferative and membranous lupus nephritis and refractory proteinuria. Hawaii J Med Public Health 2013; 72 (9 Suppl 4) : 18-23.

64. Merrill JT, Neuwelt CM, Wallace DJ, Shanahan JC, Latinis KM, Oates JC, et al. Efficacy and safety of rituximab in moderately-to-severely active systemic lupus erythematosus: The randomized, double-blind, phase II/III systemic lupus erythematosus evaluation of rituximab trial. Arthritis Rheum 2010; 62 : 222-33.

65. Reddy V, Jayne D, Close D, Isenberg D. B-cell depletion in SLE: Clinical and trial experience with rituximab and ocrelizumab and implications for study design. Arthritis Res Ther 2013; 15 (Suppl 1) : S2.

66. Wallace DJ, Kalunian K, Petri MA, Strand V, Houssiau FA, Pike M, et al. Efficacy and safety of epratuzumab in patients with moderate/severe active systemic lupus erythematosus: Results from EMBLEM, a phase IIb, randomised, double-blind, placebo-controlled, multicentre study. Ann Rheum Dis 2014; 73 : 183-90.

67. Navarra SV, Guzmán RM, Gallacher AE, Hall S, Levy RA, Jimenez RE, et al. Efficacy and safety of belimumab in patients with active systemic lupus erythematosus: A randomised, placebo-controlled, phase 3 trial. Lancet 2011; $377:$ 721-31.

68. Merrill JT, Burgos-Vargas R, Westhovens R, Chalmers A, D'Cruz D, Wallace DJ, et al. The efficacy and safety of abatacept in patients with non-life-threatening manifestations of systemic lupus erythematosus: Results of a twelve-month, multicenter, exploratory, phase IIb, randomized, double-blind, placebo-controlled trial. Arthritis Rheum 2010; $62: 3077-87$.

69. Ginzler EM, Wax S, Rajeswaran A, Copt S, Hillson J, Ramos E, et al. Atacicept in combination with MMF and corticosteroids in lupus nephritis: Results of a prematurely terminated trial. Arthritis Res Ther 2012; 14 : R33. 